\title{
A Type of Hysteretic Nonlinear Model of Piezoelectric Sensor
}

\author{
Qing-xin Zhang ${ }^{1}$, Zhi-wen $\mathrm{Zhu}^{2}$, and Xin-miao $\mathrm{Li}^{1{ }^{1 *}}$ \\ ${ }^{1}$ Beijing institute of structure and environment engineering, 1 Nan Da Hong Men Road, Beijing \\ 100076, P.R.China \\ ${ }^{2}$ Tianjin key laboratory of nonlinear dynamics and control, 92 Weijin Road, Tianjin 300072, \\ P.R.China
}

\begin{abstract}
In this paper, a type of hysteretic constitutive model of piezoelectric sensor was proposed. Nonlinear differential terms were developed to express the hysteresis phenomena of displacement-voltage relationship of piezoelectric ceramics. Based on the model, the dynamic model of a PZT sensor was established. The nonlinear dynamic behaviours of a PZT sensor were analyzed, and the relationship between the outside excitation and the voltage of a PZT sensor was obtained. Theoretical results show that there are many kinds of frequencies in the output voltage, which are caused by the PZT's hysteresis characteristics.
\end{abstract}

\section{Introduction}

Piezoelectric ceramics is a kind of smart material, which has high control accuracy and high response speed [1]. Piezoelectric sensors are widely used in engineering. The piezoelectric ceramics used widely is PZT. Usually, piezoelectric sensor is regarded as linear [2]. In fact, all kinds of piezoelectric ceramics have the hysteretic characteristics more or less [3]. To obtain the accurate response of piezoelectric sensor, an accurate physical model of piezoelectric ceramics is necessary.

Due to PZT's hysteretic characteristics, many PZT's constitutive model is equations with piecewise functions [4]. Indeed, equations with piecewise functions can express the hysteresis. However, the dynamic model of PZT structures is usually the differential equations, and the modern mathematics methods can't deal with the differential equations with piecewise functions [5]. It means that the common PZT's constitutive model can't be analysed by theory. In this paper, nonlinear differential terms were developed to describe the hysteresis phenomena of PZT's displacement-voltage curves. The physical model of a PZT sensor was established, and its nonlinear dynamic characteristics were analysed. Finally, the relationship between the outside excitation and the output voltage of PZT sensor was obtained.

\section{PZT Model}

\footnotetext{
*Corresponding author: 1xmtju@163.com
} 

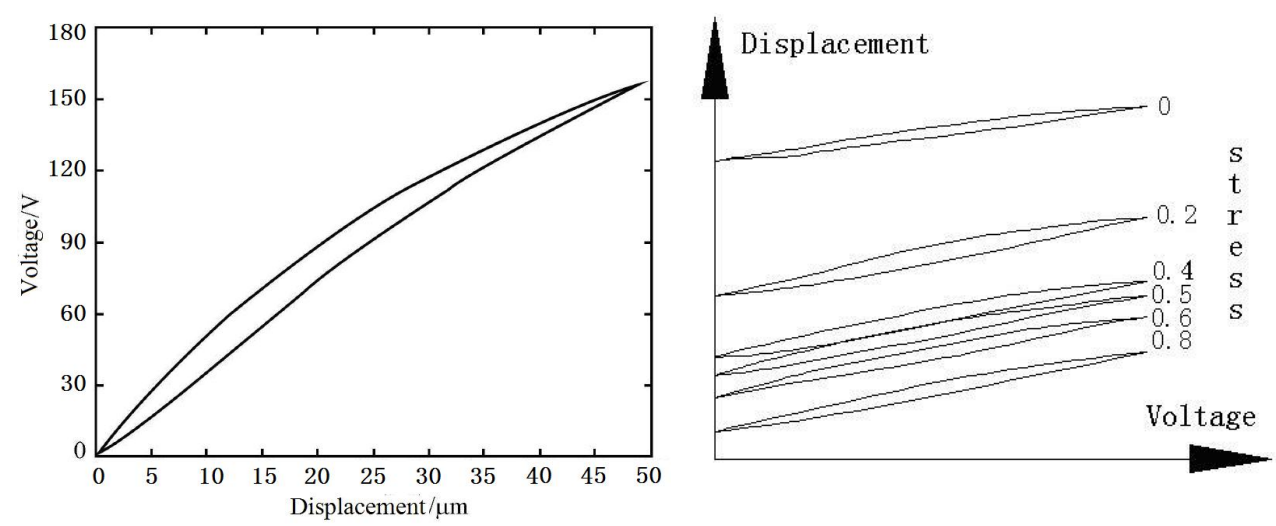

Fig. 1. PZT's displacement-voltage curves Fig. 2. Voltage-displacement curves with pre-stress

The PZT's displacement-voltage curves were in Fig.1. The relationship between strain and voltage can be shown as follows:

$$
U=\phi \varepsilon+\chi \varepsilon^{2}+\alpha \varepsilon^{3}+\beta\left(\rho \varepsilon-\varepsilon^{2}\right) \dot{\varepsilon}
$$

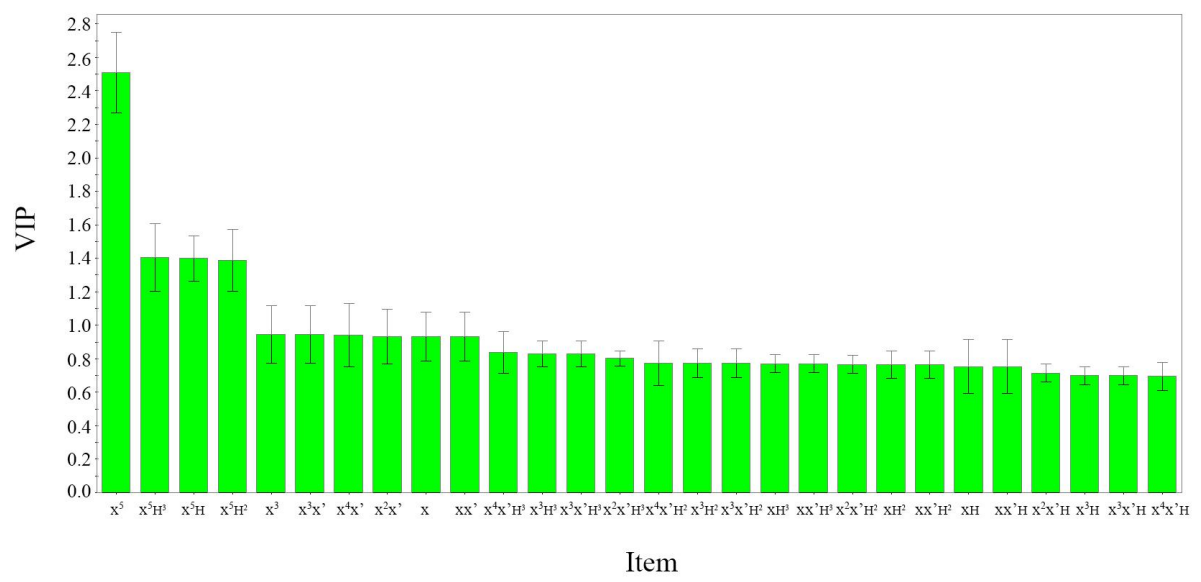

Fig. 3. Results of principal component analysis

In fact, piezoelectric layer was pre-tightened in the piezoelectric sensor, and the stress $\sigma$ will influence the PZT's strain, which is shown in Fig.2. The results of principal component analysis were in Fig.3, and the VIP values of every term were in Tab.1. Thus, the reduced constitutive model is:

$$
U=a_{0}+a_{1} \varepsilon+a_{2} \varepsilon \dot{\varepsilon}+a_{3} \varepsilon^{2} \dot{\varepsilon}=a_{1} \varepsilon+a_{2} \varepsilon \dot{\varepsilon}+a_{3} \varepsilon^{2} \dot{\varepsilon}+a_{4} \sigma+a_{5} \sigma^{2}+a_{6} \sigma^{3}
$$

where $a_{i}$ are the coefficients of terms and can be obtained in fitting method, $a_{0}=a_{4} \sigma+a_{5} \sigma^{2}+a_{6} \sigma^{3}$ was constant term which was caused by pre-stress $\sigma$. Especially, $a_{1}$ is the linear coefficient of strain $\varepsilon$, which can be shown in other physical parameters. 
Table 1. VIP of each term

\begin{tabular}{|c|c|c|c|}
\hline Term & VIP & Term & VIP \\
\hline$\varepsilon$ & 1.20443 & $\varepsilon^{2} \cdot \dot{\varepsilon} \cdot \sigma$ & 0.655907 \\
\hline$\varepsilon^{2}$ & 0.844829 & $\varepsilon \cdot \sigma^{2}$ & 0.765879 \\
\hline$\varepsilon^{3}$ & 0.993686 & $\varepsilon^{2} \cdot \sigma^{2}$ & 0.655907 \\
\hline$\varepsilon \cdot \dot{\varepsilon}$ & 1.20443 & $\varepsilon^{3} \cdot \sigma^{2}$ & 0.971131 \\
\hline$\varepsilon^{2} \cdot \dot{\varepsilon}$ & 1.04483 & $\varepsilon \cdot \dot{\varepsilon} \cdot \sigma^{2}$ & 0.765879 \\
\hline$\sigma$ & 2.08006 & $\varepsilon^{2} \cdot \dot{\varepsilon} \cdot \sigma^{2}$ & 0.655907 \\
\hline$\sigma^{2}$ & 1.22828 & $\varepsilon \cdot \sigma^{3}$ & 0.948518 \\
\hline$\sigma^{3}$ & 1.30299 & $\varepsilon^{2} \cdot \sigma^{3}$ & 0.848281 \\
\hline$\varepsilon \cdot \sigma$ & 0.846878 & $\varepsilon^{3} \cdot \sigma^{3}$ & 0.819562 \\
\hline$\varepsilon^{2} \cdot \sigma$ & 0.423017 & $\varepsilon \cdot \dot{\varepsilon} \cdot \sigma^{3}$ & 0.948518 \\
\hline$\varepsilon^{3} \cdot \sigma$ & 0.809895 & $\varepsilon^{2} \cdot \dot{\varepsilon} \cdot \sigma^{3}$ & 0.848281 \\
\hline$\varepsilon \cdot \dot{\varepsilon} \cdot \sigma$ & 0.846878 & & \\
\hline
\end{tabular}

The charge of piezoelectric patch $Q$ is

$$
Q=\iint D_{3} d y d z=D_{3} A
$$

where $D_{3}$ is the electric displacement, $A$ is the area of piezoelectric patch.

Considering that $D_{3}=d_{33} T_{3}, T_{3}=\frac{k_{2} x_{2}}{A}, x_{2}=h \varepsilon$, we obtained:

$$
Q=d_{33} T_{3} A=d_{33} k_{2} x_{2}=d_{33} k_{2} h \varepsilon
$$

where $d_{33}$ is the piezoelectric strain constant, $T_{3}$ is the stress in $\mathrm{X}$ direction, $h$ is the thickness of piezoelectric patch.

Thus,

$$
U=\frac{Q}{C}=\frac{d_{33} k_{2} h \varepsilon}{C}=\frac{d_{33} k_{2} h \varepsilon}{\frac{\varepsilon_{0} \varepsilon_{r} A}{h}}=\frac{d_{33} h^{2} k_{2}}{\varepsilon_{0} \varepsilon_{r} A} \varepsilon=a_{1} \varepsilon
$$

where $U$ is the voltage, $C$ is the capacitor, $C=\frac{\varepsilon_{0} \varepsilon_{r} A}{h}, \varepsilon_{0}$ is the vacuum permittivity, $\varepsilon_{r}$ is the relative dielectric constant of PZT.

From Eq.6, we obtained

$$
a_{1}=\frac{d_{33} h^{2} k_{2}}{\varepsilon_{0} \varepsilon_{r} A}
$$

Eq. 7 can be used to examine the accuracy of fitting result. From Figs. 4 and 5, we can see that the constitutive model has good accuracy. 


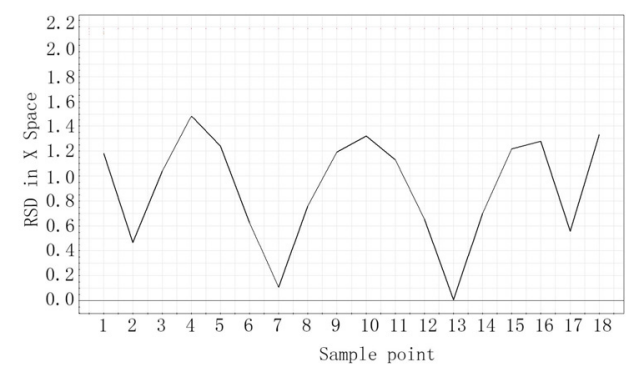

Fig. 4. Results of DModX

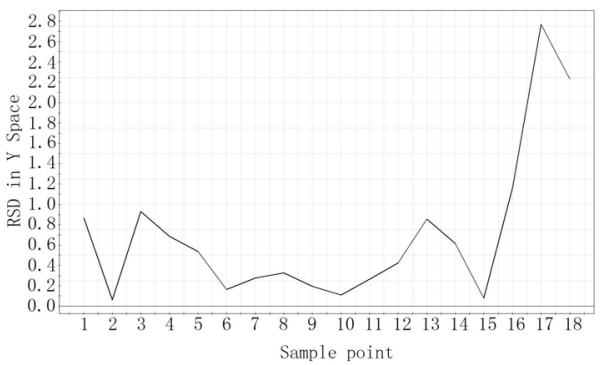

Fig. 5. Results of DModY

\section{Modeling of Piezoelectric Sensor}

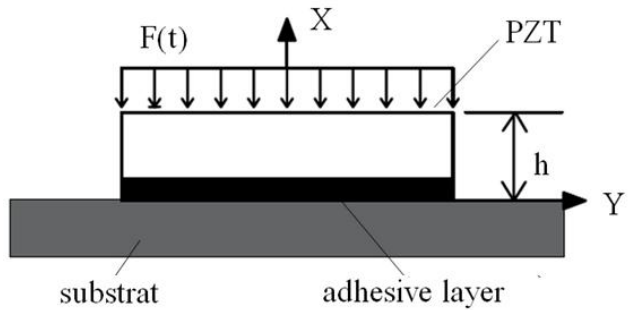

Fig. 6. Structure of piezoelectric sensor

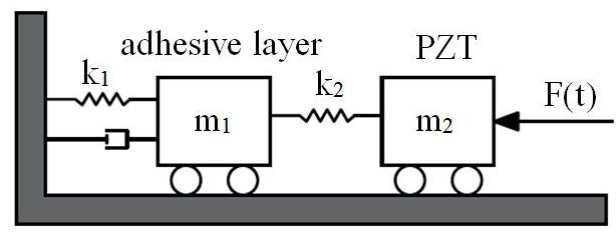

Fig. 7. Physical model of piezoelectric sensor

The structure of a piezoelectric sensor was shown in Fig.6, where PZT was glued with the substrate. The model of a piezoelectric sensor was in Fig.7, where $m_{1}$ is mass of adhesive layer, $m_{2}$ is mass of PZT, $k_{1}$ is equivalent connection stiffness of adhesive layer, $k_{2}$ is stiffness of PZT, $c_{1}$ is damping of adhesive layer, $F(t)$ is outside perturb. The mathematic model of piezoelectric sensor is:

$$
\left[\begin{array}{cc}
m_{1} & 0 \\
0 & m_{2}
\end{array}\right]\left(\begin{array}{l}
\ddot{x}_{1} \\
\ddot{x}_{2}
\end{array}\right)+\left[\begin{array}{cc}
c_{1} & 0 \\
0 & 0
\end{array}\right]\left(\begin{array}{l}
\dot{x}_{1} \\
\dot{x}_{2}
\end{array}\right)+\left[\begin{array}{cc}
k_{1}+k_{2} & -k_{2} \\
-k_{2} & k_{2}
\end{array}\right]\left(\begin{array}{l}
x_{1} \\
x_{2}
\end{array}\right)=\left(\begin{array}{c}
0 \\
F(t)
\end{array}\right)
$$

where $x_{1}$ is the deformation of adhesive layer, and $x_{2}$ is the deformation of PZT. To harmonic exciation,

$$
x_{2}=h \varepsilon=\frac{\sqrt{\left[k_{2}\left(m_{1}+m_{2}\right)-\omega^{2} m_{1} m_{2}\right]^{2}+\omega^{2} c_{1}^{2}\left(m_{1}+m_{2}\right)^{2}}}{\sqrt{a^{2}+b^{2}}} \bar{F} \cos \omega t
$$

where $x_{2}$ is displacement of piezoelectric patch in $\mathrm{X}$ direction,

$$
\begin{aligned}
& a=\omega^{4} m_{1} m_{2}-\omega^{2}\left(2 k_{2} m_{1}+k_{1} m_{2}+k_{2} m_{2}\right)+2 k_{2}\left(k_{1}+k_{2}\right) \\
& b=\omega^{2} c_{1}\left(m_{1}+m_{2}\right)+\omega c_{1}\left(3 k_{2}-k_{1}\right)
\end{aligned}
$$

Let

$$
n_{1}=\frac{\sqrt{\left[k_{2}\left(m_{1}+m_{2}\right)-\omega^{2} m_{1} m_{2}\right]^{2}+\omega^{2} c_{1}^{2}\left(m_{1}+m_{2}\right)^{2}}}{\sqrt{a^{2}+b^{2}}}
$$


Thus,

$$
\begin{aligned}
U & =a_{0}+a_{1} \varepsilon+a_{2} \varepsilon \dot{\varepsilon}+a_{3} \varepsilon^{2} \dot{\varepsilon} \\
& =a_{0}+\frac{a_{1} n_{1}}{h} \bar{F} \cos \omega t+\frac{a_{3} n_{1}^{3} \omega}{4 h^{3}} \bar{F}^{3} \sin \omega t-\frac{a_{2} n_{1}^{2} \omega}{2 h^{2}} \bar{F}^{2} \sin 2 \omega t+\frac{a_{3} n_{1}^{3} \omega}{4 h^{3}} \bar{F}^{3} \sin 3 \omega t \\
& =a_{0}+U_{0}+U_{1}
\end{aligned}
$$

where

$$
\begin{aligned}
& U_{0}=\frac{a_{1} n_{1}}{h} \bar{F} \cos \omega t \\
& U_{1}=\frac{a_{3} n_{1}^{3} \omega}{4 h^{3}} \bar{F}^{3} \sin \omega t-\frac{a_{2} n_{1}^{2} \omega}{2 h^{2}} \bar{F}^{2} \sin 2 \omega t+\frac{a_{3} n_{1}^{3} \omega}{4 h^{3}} \bar{F}^{3} \sin 3 \omega t .
\end{aligned}
$$

Eq.10 can be used in design of piezoelectric sensor:

1) $a_{0}=a_{4} \sigma+a_{5} \sigma^{2}+a_{6} \sigma^{3}$ was the constant term which was caused by stress $\sigma$;

2) $U_{0}=\frac{a_{1} n_{1}}{h} F=\frac{a_{1} n_{1}}{h} \bar{F} \cos \omega t$. It means that $U_{0}$ is linear with $F$;

3) $U_{1}$ was the error, which should be eliminated. In engineering, this term can be reduced by adjusting the parameters or active control.

\section{Conclusion}

In this paper, a type of hysteretic constitutive model of piezoelectric sensor was proposed. Nonlinear differential terms were developed to express the hysteresis phenomena of displacement-voltage relationship of piezoelectric ceramics. Based on the model, the dynamic model of a PZT sensor was established. The nonlinear dynamic behaviours of a PZT sensor were analysed, and the relationship between the outside excitation and the voltage of a PZT sensor was obtained. We can see that there are many kinds of frequencies in the output voltage, which are caused by the PZT's hysteresis characteristics.

\section{Acknowledgements}

The authors thank the support of Tianjin RPAFAT through Grant No. 16JCYBJC18800, and Cast-Bisee 511 Program through Grant No. CAST-BISEE2017-006.

\section{References}

[1] N.W. Hagood, W.H. Chung and A.V. Journal of Intelligent Material Systems and Structures 1 (1990).

[2] P.Ge, M. Precision Engineering 20 (1997).

[3] M.H. Shen. Computers and Structures 57 (1995).

[4] L.N. Sun, C.H. Ru and W.B. Rong. Journal of Micromechanics and Microengineering 14 (2004).

[5] M. Deshpande, L. Saggere. Sensors and Actuators A-Physical 136 (2007).

[6] C.H. Ru, L.G. Chen and B. Shao. Control Engineering Practice 17 (2009). 